\title{
The Number of Extreme Triples of a Planar Point Set*
}

\author{
E. A. $\operatorname{Ramos}^{\dagger}$ \\ Department of Computer Science, University of Illinois at Urbana-Champaign, \\ Urbana, IL 61801, USA
}

\begin{abstract}
An extreme triple or 3-set of a finite set $S$ in the plane is a subset of $S$ of size 3 of the form $S \cap h$, for some half-plane $h$. We establish an upper bound $\lfloor 11 n / 6\rfloor+1$ for the number of extreme triples of any $S$ with $|S|=n \geq 10$. This almost matches the known lower bound $[11 n / 6]$.
\end{abstract}

\section{Introduction}

Let $S$ be a set of $n$ points in $\mathbf{R}^{2}$. For a half-plane $h, S^{\prime}=S \cap h$ is called a $k$-set of $S$ where $k=\left|S^{\prime}\right|$. Without loss of generality we can consider only half-planes determined by lines that do not contain any of the points in $S$. Let $e_{k}(S)$ denote the number of $k$-sets realized by $S$, and define $e_{k}(n)=\max \left\{e_{k}(S): S \subseteq \mathbf{R}^{2},|S|=n\right\}$ for $1 \leq k \leq n-1$. For the importance of these numbers in combinatorial and computational geometry, and for references, see [1] and [4]. We also say extreme pairs and extreme triples, or pairs and triples for short, instead of 2-sets and 3-sets. Edelsbrunner and Stöckl [6], [2] determined that $e_{2}(n)=\lfloor 3 n / 2\rfloor$ for $n \geq 4$, and they found a construction that shows that $e_{3}(n) \geq 11 n / 6$, for $n$ a multiple of six, which can be extended to $e_{3}(n) \geq\lfloor 11 n / 6\rfloor$ for $n \geq 15, n \neq 19$. In this note we prove a corresponding upper bound $\lfloor 11 n / 6\rfloor+1$.

As defined, $k$-sets are determined by half-planes bounded by (straight) lines, so we can say that the $k$-sets are determined by an arrangement of lines. Our upper bound is actually for $k$-sets determined in the same manner on a set of points $S$ by half-planes bounded by an arrangement of pseudolines $\mathcal{C}$, that is, a collection of topological lines in which every pair intersects exactly once at a crossing point (and no point in $S$ incident to a pseudoline in $\mathcal{C}$ ). For a point set $S$ and a pseudoline arrangement $\mathcal{C}$, let $g_{k}(S, \mathcal{C}$ ) be the

\footnotetext{
* This research was supported by the National Science Foundation under Grant CCR-9118874.

Current address: DIMACS Center, Rutgers University, P.O. Box 1179, Piscataway, NJ 08855-1179. ramose@dimacs.rutgers.edu.
} 
number of $k$-sets determined by $\mathcal{C}$ on $S$, let $g_{k}(S)$ be the maximum of $g_{k}(S, \mathcal{C})$ over all arrangements of pseudolines $\mathcal{C}$, and let $g_{k}(n)=\max \left\{g_{k}(S): S \subseteq \mathbf{R}^{2},|S|=n\right\}$. Clearly, $e_{k}(n) \leq g_{k}(n)$. There is another definition of $k$-sets, based on $k$-levels in an arrangement of pseudolines, which is equivalent to the definition based on circular sequences [1], [4]. Because of a duality principle for arrangements of pseudolines [3], the $k$-sets in both definitions are related; we do not elaborate on this relation.

Our proof of the upper bound for $g_{3}(n)$ is essentially a (somewhat tedious) case analysis of the possible configurations which concludes that the lower-bound construction is optimal. We believe that $\lfloor 11 n / 6\rfloor$ is the correct upper bound for $n \geq 7$, but we have avoided a more careful consideration of configurations with few points which would settle the matter.

There are difficulties in extending this to larger values of $k$. First, we note that $g_{k}(n) \leq$ $\left\lfloor\alpha_{k} n\right\rfloor$ where $\alpha_{k}=\sup \left\{g_{k}(m) / m: m \geq 1\right\}$. The upper bound proof for $g_{3}(n)$ is possible because the sup defining $\alpha_{3}$ is achieved and the extremal configuration is periodic. It is not clear that this is the case in general. Second, even if this is true, the corresponding case analysis already becomes too difficult to handle for $k=4$. A possible alternative would be to devise a systematic approach that could be implemented on a computer. More importantly, the real interest is to estimate $e_{k}(n)$ and $g_{k}(n)$ for $k=\Theta(n)$.

In Section 2 we explain the approach and illustrate it by proving that $g_{2}(n) \leq\lfloor 3 n / 2\rfloor$ (essentially the proof in [2] but using our framework); Section 3 contains the proof of the upper bound for $g_{3}(n)$.

\section{Framework and Upper Bound for $\boldsymbol{g}_{2}(\boldsymbol{n})$}

Layers. For $j \geq 0$, let the $j$-layer $L_{j}$ be the boundary of the $j$-hull of $S$ and let the $j$-vertices $S_{j}$ be those points of $S$ on $L_{j}$ ( $L_{j}$ is the boundary of the convex hull of $T_{j}$ where $T_{0}=S$ and $T_{j}=S-\bigcup_{i=0}^{j-1} S_{i}$ for $j \geq 1$ ). The edges between consecutive $j$-vertices are called $j$-edges. Any line crosses $L_{j}$ twice or not at all, but crosses any $j$-edge at most once. Hence, if a $k$-set contains a $j$-vertex, then it contains an $i$-vertex for $i<j$. Thus, we can assume that $S_{j}=\emptyset$ for $j \geq k$.

A similar situation can be obtained when we consider $k$-sets determined by an arrangement of pseudolines. Let the layers $L_{0}, L_{1}, \ldots, L_{k-1}$ be nested closed simple curves in order from outside (for example, concentric circles), and let $p$ be a point inside $L_{k-1}$. The $j$-vertices and $j$-edges are defined as before. The $k$-sets are determined by an arrangement of curves $\mathcal{C}$ such that:

(i) Each $C \in \mathcal{C}$ is topologically a line that starts and ends outside $L_{0}$.

(ii) Each pair $C, C^{\prime} \in \mathcal{C}$ intersects once at a crossing point.

(iii) Each $C \in \mathcal{C}$ crosses each $L_{j}$ twice or not at all but not in points of $S_{j}$ and not more than once in any $j$-edge.

The set of points of $S$ in the half-plane not containing $p$ bounded by a pseudoline $C \in \mathcal{C}$ is a (pseudo) $k$-set if its cardinality is $k$. Note that if a $k$-set contains a $j$-vertex, then it also contains an $i$-vertex for $i<j$.

We need to argue that the curves $L_{j}$ and the point $p$ exist for a given arrangement of pseudolines $\mathcal{C}$ determining $k$-sets: $p$ is guaranteed to exist for $n \geq 3 k+1$ by Helly's 
theorem. To obtain the curves $L_{j}$, start with a large pseudocircle $\mathbf{C}$ that contains the point set $S$ and intersects each $C \in \mathcal{C}$ twice; then sweep with $\mathbf{C}$ over $\mathcal{C}$ toward $p$ (in [5] it is shown that this is possible). Let $T_{0}=S$ and $T_{j}=S-\bigcup_{i=0}^{j-1} S_{i}$ for $j \geq 1$. Sweeping as far as possible but without going beyond any of the points in $T_{j}$ results in $L_{j}$; $S_{j}$ is the set of points of $S$ on $L_{j}$. Under the assumption that each point in $S$ is in some $k$-set determined by a curve in $\mathcal{C}$, then $S=\bigcup_{j=0}^{k-1} S_{j}$.

By removing $p$, we can view the curves $L_{j}$ as nonintersecting curves on a cylindrical surface wrapping around once. In our figures we draw the curves $L_{j}$ as horizontal lines in the plane and assume that they wrap around and close outside of the window in the figure; thus, the pseudolines in $\mathcal{C}$ start and end below $L_{0}$, and the half-planes determining $k$-sets are those below the pseudolines.

Conventions. For the purpose of the presentation, a $j$-vertex is labeled with a letter with subscript $j$. The labels of consecutive $j$-vertices are consecutive letters from the end of the English alphabet. For example, the vertex $u_{j}$ is on $L_{j}$ and its neighbors to the left and right are $t_{j}$ and $v_{j}$. Greek letters are used when we do not want to make an implicit statement about the relative positions, and subscripts omitted when its hull index is undetermined. Pairs and triples are written as $(\alpha \beta)$ and $(\alpha \beta \gamma)$ where $\alpha, \beta, \gamma$ are vertices. For the purpose of referring to figures, the vertices with label $u$ (in each hull) are drawn as a square (rather than a circle).

Proof Structure. The goal is to prove by induction that $g_{k}(n) \leq q n / p+C$ where $C$ is a constant. For the basis, we check exhaustively all the possible configurations for point sets with less than some $D$ points and find that $g_{k}(n) \leq q n / p+C$ for $n<D$. For the inductive step, we consider an arbitrary configuration with $n \geq D$ vertices and show that either it is not maximal ( $k$-sets can be added) or it is reducible, that is, it is possible to modify the point set and arrangement locally, so that $p^{\prime}$ vertices are removed, and the number of $k$-sets is reduced by at most $q^{\prime}$ (some $k$-sets disappear, some new ones are introduced) with $p^{\prime}$ and $q^{\prime}$ satisfying $q^{\prime} / p^{\prime} \leq q / p$. This is called a $\left(p^{\prime}, q^{\prime}\right)$ reduction. Thus, we have

$$
\begin{aligned}
g_{k}(n) & \leq g_{k}\left(n-p^{\prime}\right)+q^{\prime} \leq q\left(n-p^{\prime}\right) / p+C+q^{\prime} \\
& =q n / p-q p^{\prime} / p+q^{\prime}+C \leq q n / p+C,
\end{aligned}
$$

where we have used the inductive hypothesis for the second inequality, and $q^{\prime} / p^{\prime} \leq q / p$ for the last one.

For convenience of exposition, the inductive step (the fact that a maximal configuration with $n \geq D$ can be reduced) is presented as a sequence of properties that a maximal irreducible configuration must satisfy. The conclusion that no maximal configuration with $n \geq D$ is irreducible proves the inductive step ( $D$ is the smallest $n$ for which the reductions apply). The proofs of these properties consider a window of the configuration consisting of some constant number of consecutive vertices in each of the layers, and assumes that the pseudolines that determine $k$-sets on the vertices in the window do not wrap around. See Fig. 1. This is easily justified for $k=2$ but more care is needed for $k=3$. Within a window, we can regard the vertices in the $j$-layer as linearly ordered from left to right. Two possible $k$-sets (sets of points of cardinality $k$ ) are said to conflict if there are no curves that determine them and satisfy conditions (i)-(iii) above. 


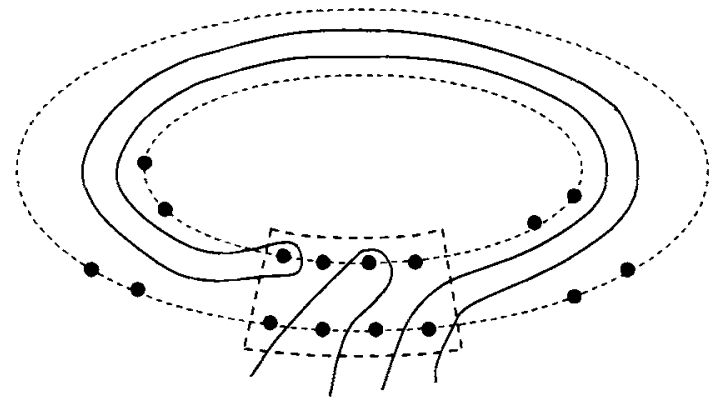

Fig. 1. Pseudoline wrapping around.

Upper Bound for $g_{2}(n)$. Only two types of extreme pairs are possible: An H-pair is of the form $\left(u_{0} v_{0}\right)$, and a V-pair is of the form $\left(u_{0} u_{1}\right)$. The initials $\mathbf{H}$ and $\mathbf{V}$ stand for horizontal and vertical. See Fig. 2. Within a window, two $V$-pairs $\left(\alpha_{0} \alpha_{1}\right)$ and $\left(\beta_{0} \beta_{1}\right)$ are in conflict if either

(i) $\alpha_{1}=\beta_{1}$ and $\alpha_{0}$ and $\beta_{0}$ are not adjacent, or

(ii) $\alpha_{0}$ is to the left of $\beta_{0}$ and $\alpha_{1}$ is to the right of $\beta_{1}$, or vice versa (this assumes no wrapping around).

The goal is to prove that the number of pairs is at most $3 n / 2+C$, so $(1,1)$ and $(2,3)$ reductions are acceptable. We have the following sequence of observations about an irreducible configuration assuming that there is no wrapping around:

(1) If $\left(u_{0}, \alpha_{1}\right)$ and $\left(v_{0}, \beta_{1}\right)$ are V-pairs, then $\alpha_{1}$ is not to the right of $\beta_{1}$ : otherwise there is a conflict of type (ii).

(2) A 1-vertex belongs to at most two $\mathbf{V}$-pairs (except if $n=4$ ): otherwise there is a conflict of type (i).

(3) A 1-vertex belongs to at least two $\mathbf{V}$-pairs: otherwise it can be removed, a $(1,1)$ reduction.

(4) A 0-vertex belongs to at least one V-pair: otherwise it can be removed, a $(1,1)$ reduction.

(5) Two adjacent 0 -vertices belong to $\mathbf{V}$-pairs with a common 1-vertex: otherwise the 0 -edge can be "contracted", a $(1,1)$ reduction.

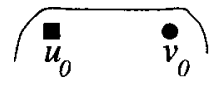

H-pair

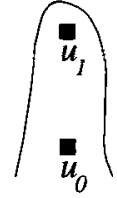

V-pair



type (i) conflict



type (ii) conflict

Fig. 2. Pairs and conflicts. 


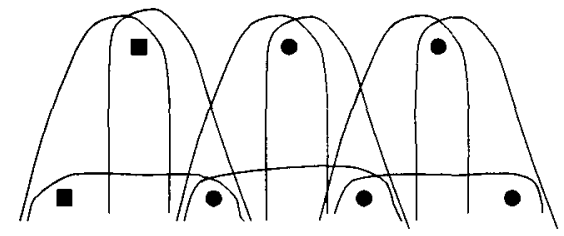

Fig. 3. Optimal configuration for $k=2$.

(6) A 0-edge corresponds to an H-pair: otherwise it can be added without introducing any conflict.

Finally, the only configuration possible where neither of these reductions apply is one in which each 0-edge forms an $\mathbf{H}$-pair and a corresponding 1-vertex forms $\mathbf{V}$-pairs with each of the two 0 -vertices of the 0 -edge. See Fig. 3. In this configuration, a 0 -edge can be "contracted" and its corresponding 1-vertex removed, resulting in a $(2,3)$ reduction. Finally, the possibility of a pseudoline wrapping around only affects a reduction when as a result two pseudolines would intersect twice. By considering the small configurations for which this happens, it is found that $\lfloor 3 n / 2\rfloor$ is a tight upper bound.

\section{Upper Bound for $g_{3}(n)$}

\subsection{Types of Triples}

Four types of triples are possible: An H-triple is of the form $\left(u_{0} v_{0} w_{0}\right)$, a V-triple is of the form $\left(u_{0} u_{1} u_{2}\right)$, a $\mathbf{D}$-triple is of the form $\left(u_{0} v_{0} u_{1}\right)$, and an $\mathbf{N}$-triple is of the form $\left(u_{0} u_{1} v_{1}\right)$. The initials $\mathbf{H}, \mathbf{V}, \mathbf{D}$, and $\mathbf{N}$ stand for horizontal, vertical, delta, and nabla, respectively. See Fig. 4.

Assuming no wrapping around, the triples of types $\mathbf{D}$ and $\mathbf{N}$ conflict in cases similar to those of the pairs, as illustrated in Fig. 5.
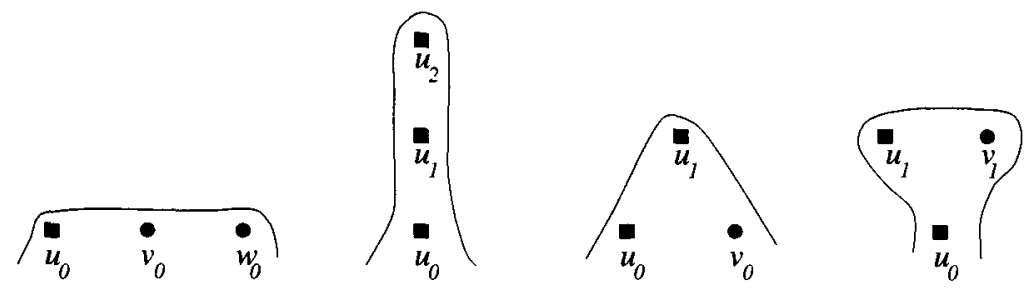

Fig. 4. Triples $\mathbf{H}, \mathbf{V}, \mathbf{D}$, and $\mathbf{N}$. 

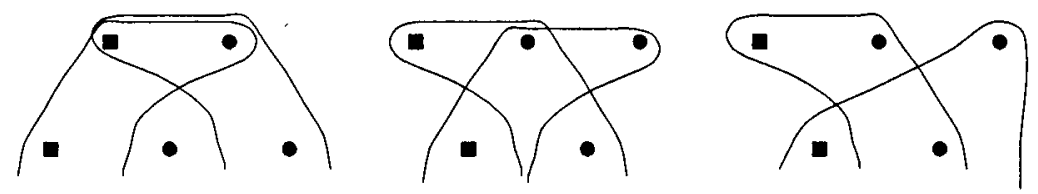

Fig. 5. Some conflicts between triples.

\subsection{Lower-Bound Construction}

The construction in [6] and [2] played an important role in devising the upper-bound argument; in fact, the proof aims to show that the construction is optimal. The basic configuration is a sequence of six points. Figure 6 shows eleven straight lines that determine eleven extreme triples on the six points of a basic configuration. Note that these eleven triples use the first two points of the next basic configuration. Figure 7 shows the eleven triples of the basic configuration using our framework. For $n=6 m$, $m \geq 2$, the construction consists in repeating $m$ times the basic configuration wrapping around (wrapping around one basic configuration results in conflicting triples). This can be extended to $n$ not a multiple of six by removing 2-vertices (note that for $n \bmod 6=1,2,3,4,5,\lfloor 11(n \bmod 6) / 6\rfloor=1,3,5,7,9$, respectively). For $n \geq 7$, this fails for $n=7,8,9,13,14,19$. For $n=7,13,19$, in the constructions for $n=5,11,17$, insert two extra vertices as shown in Fig. 8(a) which introduce three additional triples. For $n=8,9,14$, in the construction for $n=5,6,11$, insert three extra points as shown in Fig. 8(b) which introduce five additional triples. This shows that $g_{3}(n) \geq\lfloor 11 n / 6\rfloor$ for $n \geq 7$.

The basic construction in Fig. 6 can be modified by an affine transformation so that it can be repeated around a circle $m$ times for $m \geq 3$, wrapping around. By removing 2 -vertices this shows the second part of the following theorem.

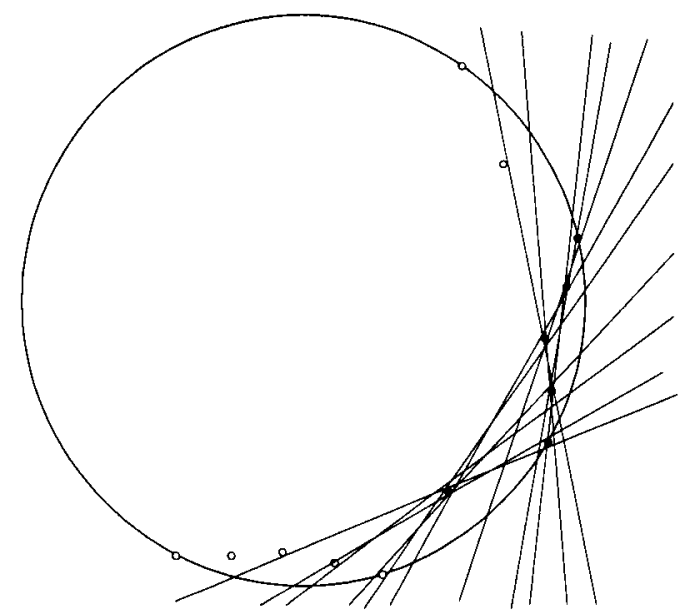

Fig. 6. Lower-bound construction. 


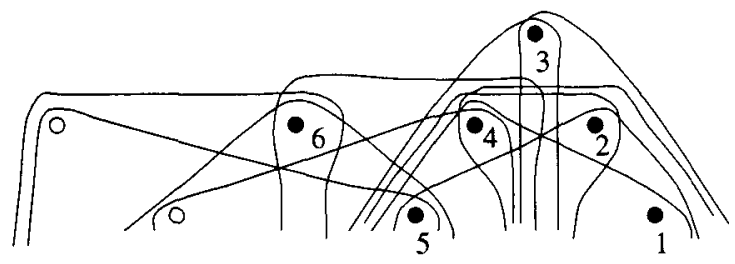

Fig. 7. Lower-bound construction in our framework.

\section{Theorem 3.1.}

(i) $g_{3}(n) \geq\lfloor 11 n / 6\rfloor$ for $n \geq 7$.

(ii) $e_{3}(n) \geq\lfloor 11 n / 6\rfloor$ for $n \geq 15, n \neq 19$.

\subsection{Proof of the Upper Bound}

Reductions. In general, a reduction consists in removing some vertices which results in some triples lost, possibly adding other vertices (with a net decrement in the number), and then introducing some new triples that become possible (this requires some local modification of the arrangement). We mention some examples of reductions. In a contraction of an edge $\alpha \beta$, vertices $\alpha$ and $\beta$ and all triples containing either of them are removed, a new vertex $\gamma$ is introduced together with as many as possible of the triples that contained either $\alpha$ or $\beta$ but not both, now substituted with $\gamma$. See Fig. 12 for an example. We also contract two or three consecutive edges into one, this requires removing all but the extreme vertices in those edges. See Fig. 22 for an example (where vertex $u_{1}$ has also been removed). A collapse is performed when the same configuration appears repeated consecutively, one of the copies is removed. See Fig. 14 for an example. In some reductions, as in Fig. 17, after removing some vertices (the black ones), four vertices (the white ones in layers 0 and 1) become adjacent and new triples can be introduced.

Cutting Open the Arrangement. For $k=3$, it becomes more difficult to consider small configurations for which the observations do not apply, because of pseudolines wrapping around. We get around by "cutting" the cylindrical surface and the arrangement so that the layers $L_{j}$ become horizontal lines in the plane (but now they are not assumed to wrap around and close), the pseudolines begin and end below the lowest layer line, and the half-spaces determining $k$-sets are those below the pseudolines. We define $g_{k}^{\prime}(n)$ in this setting analogously to $g_{k}(n)$. The connection between $g_{k}(n)$ and $g_{k}^{\prime}(n)$ depends on

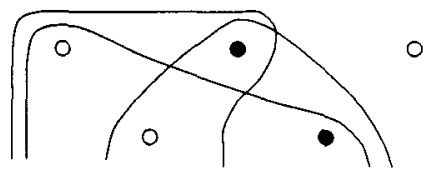

(a)

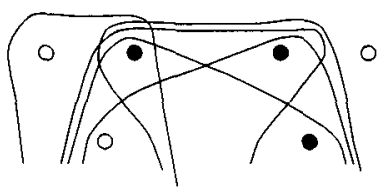

(b)

Fig. 8. Extending the lower bound to nonmultiples of six. 

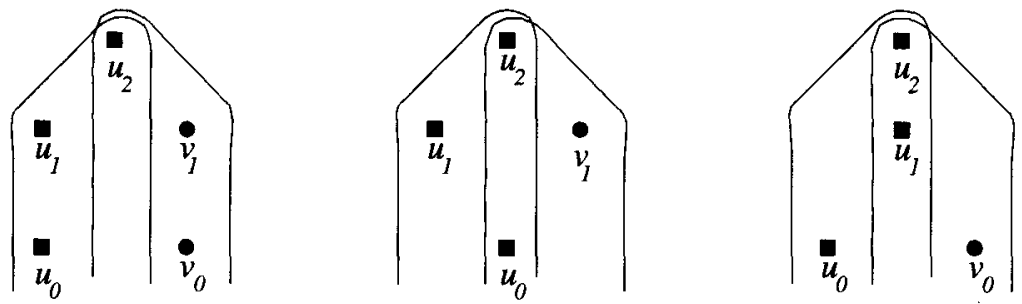

Fig. 9. Configurations $\langle 2,2\rangle,\langle 1,2\rangle$, and $\langle 2,1\rangle$.

the procedure for cutting open the arrangement; this is dealt with in the final argument. All the observations below are for this setting in which there is no wrapping around. Unfortunately, in this way we do not obtain a tight bound (assuming that the additive constant is actually zero).

Observations. The proof follows the outline described in the previous section. We have a sequence of observations that apply, given the assumption of no wrapping around, to an irreducible maximal configuration (this is just an alternative way of presenting the inductive step of the proof). It is shown that a configuration which does not have the claimed property can be locally modified resulting in a $\left(p^{\prime}, q^{\prime}\right)$ reduction for $p^{\prime}, q^{\prime}$ with $q^{\prime} / p^{\prime} \leq 11 / 6$. In particular, any $\left(p^{\prime}, q^{\prime}\right)$ reduction with $p^{\prime} \leq 6$ and $q^{\prime}<2 p^{\prime}$ is acceptable. In the proofs, previous observations are often used without mention.

Observation 3.2. A 2-vertex $u_{2}$ belongs to exactly two $\mathbf{V}$-triples and this can happen in only three possible configurations (see Fig. 9):

(i) A $\langle 2,2\rangle$-configuration $\left[u_{0} v_{0}, u_{1} v_{1}, u_{2}\right]$ which consists of triples $\left(u_{0} u_{1} u_{2}\right)$ and $\left(v_{0} v_{1} u_{2}\right)$.

(ii) $A\langle\mathbf{1}, 2\rangle$-configuration $\left[u_{0}, u_{1} v_{1}, u_{2}\right]$ which consists of triples $\left(u_{0} u_{1} u_{2}\right)$ and $\left(u_{0} v_{1} u_{2}\right)$.

(iii) $A\langle 2, \mathbf{1}\rangle$-configuration $\left[u_{0} v_{0}, u_{1}, u_{2}\right]$ which consists of triples $\left(u_{0} u_{1} u_{2}\right)$ and $\left(v_{0} u_{1} u_{2}\right)$.

Proof. Consider the V-triples that contain the 2-vertex $u_{2}$. If $u_{2}$ is in a $\mathbf{V}$-triple with $\alpha_{1}$ and in a $V$-triple with $\beta_{1}$ then $\alpha_{1}$ and $\beta_{1}$ must be adjacent. Similarly for 0 -vertices in $\mathrm{V}$-triples with $u_{2}$. So let $u_{0}, v_{0}, u_{1}, v_{1}$ be the vertices that may belong to a $\mathrm{V}$-triple together with $u_{2}$. This limits the number of $V$-triples of $u_{2}$ to four. However, two of them conflict: $\left(u_{0} v_{1} u_{2}\right)$ and $\left(v_{0} u_{1} u_{2}\right)$; see Fig. 10(i). Thus, $u_{2}$ can belong to at most three V-triples: $\left(u_{0} u_{1} u_{2}\right),\left(v_{0} v_{1} u_{2}\right)$, and $\tau_{1}=\left(v_{0} u_{1} u_{2}\right)$ (or the symmetric one); see Fig. 10(ii) (we call this a $\langle 2,2\rangle^{\prime}$-configuration). However, $\tau_{1}$ can be substituted with $\tau_{2}=\left(u_{0} v_{0} v_{1}\right)$ without changing the number of triples. The reason is that $\tau_{1}$ and $\tau_{2}$ conflict (so $\tau_{2}$ could not have been in the original configuration) and, as inspection shows, any triple that conflicts with $\tau_{2}$ (other than $\tau_{1}$ ) also conflicts with $\tau_{1}$ or with one of the $\mathbf{V}$-triples. See Fig. 10(ii). Thus we can assume that $u_{2}$ belongs to at most two $\mathrm{V}$-triples. On the other 


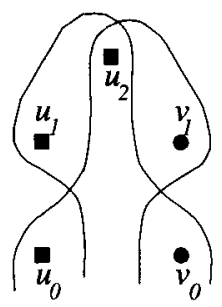

(i)

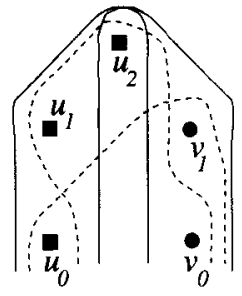

(ii)

Fig. 10.

hand if $u_{2}$ is only in one triple, then it can be removed with a loss of only one triple. Therefore the only possible cases are those listed in the statement of the observation.

\section{Observation 3.3.}

(i) A 0-edge belongs to at most one $\langle 2,2\rangle$ - or $\langle 2,1\rangle$-configuration.

(ii) A 1-edge belongs to at most one $\langle 2,2\rangle$-or $\langle\mathbf{1}, 2\rangle$-configuration.

(iii) A 1-vertex belongs to at most one $\langle 2,1\rangle$-configuration.

Proof. (i) A 2-vertex cannot be in two $\langle 2,2\rangle$ - or $\langle\mathbf{2}, \mathbf{1}\rangle$-configurations, so if $\left(u_{0} v_{0}\right)$ is in two of these configurations, then there is a pair of triples $\left(u_{0} \alpha_{1} v_{2}\right)$ and $\left(v_{0} \beta_{1} u_{2}\right)$ which are in conflict. (ii) Similarly, if $\left(u_{1} v_{1}\right)$ is in two configurations, then there is a pair of triples $\left(\alpha_{0} u_{1} v_{2}\right)$ and $\left(\beta_{0} v_{1} u_{2}\right)$ which are in conflict. (iii) Similar to (i) and (ii).

Observation 3.4. There is no pair of triples $\left(u_{0} u_{1} v_{1}\right)$ and $\left(v_{0} w_{0} u_{1}\right)$, nor the symmetric configuration.

Proof. Suppose $\tau_{1}=\left(u_{0} u_{1} v_{1}\right)$ and $\tau_{2}=\left(v_{0} w_{0} u_{1}\right)$ are in the original configuration. Then substitute $\tau_{2}$ with $\tau_{3}=\left(u_{0} v_{0} v_{1}\right) . \tau_{3}$ was not there because it conflicts with $\tau_{2}$, and inspection shows that $\tau_{3}$ introduces no conflicts. See Fig. 11(i). Note also that $\tau_{3}$ does not introduce a configuration of the type that is being avoided: this could only occur if either $\left(w_{0} u_{1} v_{1}\right)$ or $\left(t_{0} v_{1} w_{1}\right)$ (dotted in Fig. 11(ii)) is present, but they cannot as they conflict with $\tau_{1}$.

Observation 3.5. There are no $\langle 2, \mathbf{1}\rangle$-configurations.

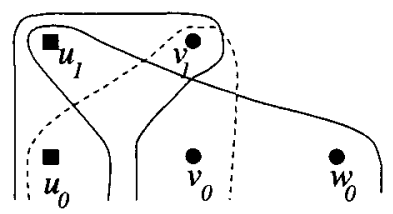

(i)

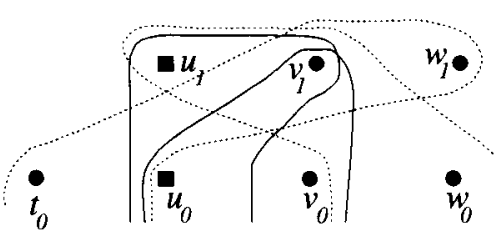

(ii)

Fig. 11. 


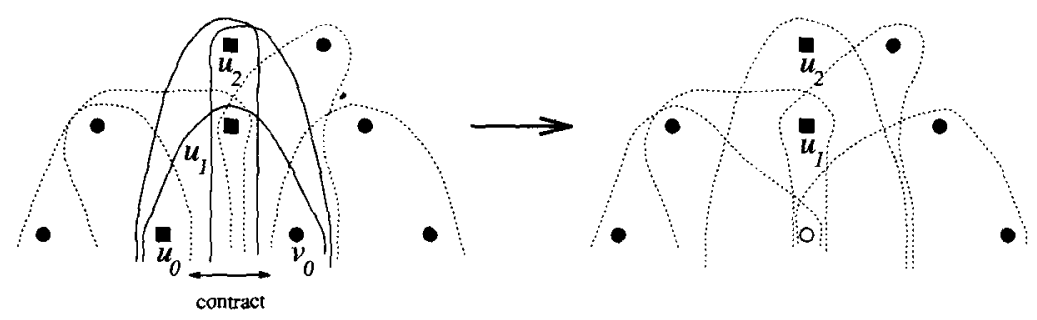

Fig. 12.

Proof. Suppose the $\langle 2,1)$-configuration $\left[u_{0} v_{0}, u_{1}, u_{2}\right]$ occurs. Then contracting $u_{0} v_{0}$ and removing $u_{2}$ results in a $(2,3)$ reduction. $\left(u_{0} v_{0} u_{1}\right)$ is the third triple that may be lost, and no other triples may be lost. Figure 12 shows the contraction. In particular, no H-triple may contain $u_{0} v_{0}$, no other D-triple may contain $u_{0} v_{0}$, and it is not possible to have two $\mathbf{N}$-triples containing the same 1 -edge and each of $u_{0}$ and $v_{0}$. No conflicts are introduced by the contraction.

Observation 3.6. There are no $\langle\mathbf{1}, 2\rangle$-configurations, and there are at most two $\mathbf{N}$ triples containing the same 0 -vertex.

Proof. First, we rule out some configurations to simplify further analysis:

(a) There are no two $(1,2)$-configurations with the same 0 -vertex $u_{0}$, adjacent as $\left[u_{0}, u_{1} v_{1}, u_{2}\right]$ and $\left[u_{0}, v_{1} w_{1}, v_{2}\right]$, or with $\mathbf{N}$-triples in between.

(b) There are no two adjacent $\langle\mathbf{1}, 2\rangle$-configurations $\left[u_{0}, u_{1} v_{1}, u_{2}\right]$ and $\left[v_{0}, v_{1} w_{1}, v_{2}\right]$.

(c) There is no $\langle\mathbf{1}, 2\rangle$-configuration $\left[u_{0}, u_{1} v_{1}, u_{2}\right]$ adjacent to a $\langle 2,2\rangle$-configuration $\left[v_{0} w_{0}, v_{1} w_{1}, v_{2}\right]$, or the symmetric configuration.

See Fig. 13. The first case is taken care with a collapse, shown in Fig. 14, and the others by contracting $u_{0} v_{0}$.

The case left is that of a $\langle 1,2\rangle$-configuration $\left[u_{0}, u_{1} v_{1}, u_{2}\right]$ which is not adjacent to any other $\langle 1,2\rangle$-configuration, and not adjacent to a $\langle 2,2\rangle$-configuration in the form ruled out above (but an adjacent $\langle 2,2\rangle$-configuration $\left[u_{0} v_{0}, v_{1} w_{1}, v_{2}\right]$ is possible, and similarly on the left). Removing $u_{2}$ and contracting $u_{1} v_{1}$ results in a $(2,3)$ reduction, except if there are two triples separated in the 0 -layer by a vertex and containing $u_{1}$ and
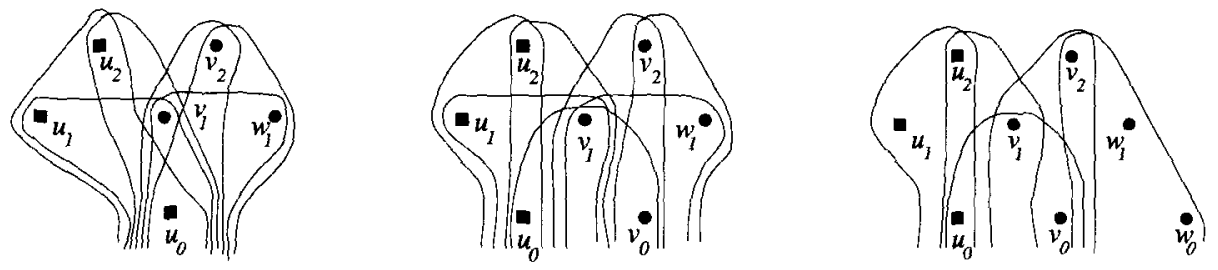

Fig. 13. 

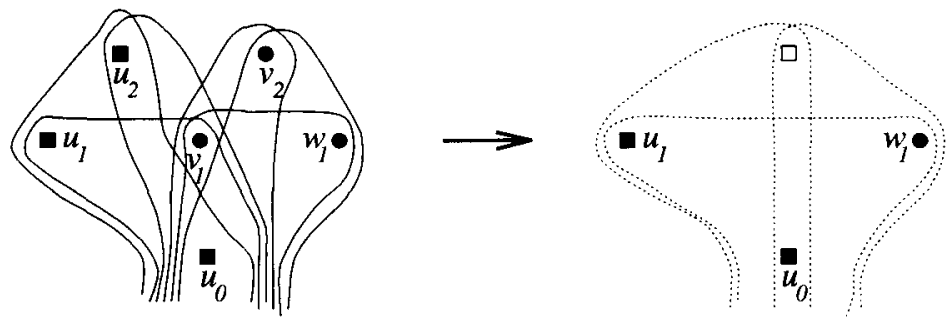

Fig. 14.

$v_{1}$, respectively (because they would conflict after the contraction). On the left side, this can be the result of either the $\mathbf{N}$-triple $\left(t_{0} t_{1} u_{1}\right)$, or the D-triple $\left(s_{0} t_{0} u_{1}\right)$, or both of them (it cannot be a V-triple because of the cases already ruled out). Similarly on the right side. If both of them are present (on the same side, say on the left) then substitute $\left(s_{0} t_{0} u_{1}\right)$ with $\left(t_{0} u_{0} t_{1}\right)$, which is shown dashed in Fig. 15.

Case (ii) only (on one side, say on the left) is taken care of by contracting $t_{0} u_{0}$, removing $u_{2}$, and contracting $u_{1} v_{1}$. See Fig. 16 . This is a $(3,5)$ reduction, since the $\mathbf{H}$-triple $\left(s_{0} t_{0} u_{0}\right)$ may be present (it is not possible to have the $\mathbf{N}$-triple $\left(u_{0} t_{1} u_{1}\right)$ because together with $\left(s_{0} t_{0} u_{1}\right)$ it forms the configuration forbidden by Observation 3.4).

Finally, case (i) only (on both sides) is taken care of by removing $u_{0}, u_{1}, v_{1}, u_{2}$ (and $t_{2}$ and/or $v_{2}$ if they are in $\langle 2,2\rangle$-configurations $\left[t_{0} u_{0}, t_{1} u_{1}, t_{2}\right]$ and $\left[u_{0} v_{0}, v_{1} w_{1}, v_{2}\right]$ ) resulting in a $(4,7)$ reduction (or $(5,9)$ or $(6,11)$, respectively). See Fig. 17 . The figure on the left shows with continuous lines the seven triples that can be lost: $\left(u_{0} u_{1} u_{2}\right),\left(u_{0} v_{1} u_{2}\right)$, $\left(u_{0} u_{1} v_{1}\right),\left(t_{0} u_{0} u_{1}\right),\left(u_{0} v_{0} v_{1}\right),\left(t_{0} t_{1} u_{1}\right),\left(v_{0} v_{1} w_{1}\right)$. The figure on the right shows the four triples that can be introduced after removing the vertices; they replace the triples $\left(u_{0} t_{1} u_{1}\right)$, $\left(u_{0} v_{1} w_{1}\right),\left(t_{0} u_{0} t_{1}\right),\left(u_{0} v_{0} w_{1}\right)$ whenever they were present (if an $\mathbf{N}$-triple is not possible after the reduction, then the corresponding triple was not present before the reduction).

\section{Observation 3.7.}

(i) If the $\mathbf{N}$-triples $\left(u_{0} u_{1} v_{1}\right)$ and $\left(v_{0} u_{1} v_{1}\right)$ occur, then the $\mathbf{D}$-triples $\left(u_{0} v_{0} u_{1}\right)$ and $\left(u_{0} v_{0} v_{1}\right)$ also occur.

(ii) If the $\langle\mathbf{2}, \mathbf{2}\rangle$-configuration $\left[u_{0} v_{0}, u_{1} v_{1}, u_{2}\right]$ occurs, then the $\mathbf{D}$-triples $\left(u_{0} v_{0} u_{1}\right)$ and $\left(u_{0} v_{0} v_{1}\right)$ also occur.

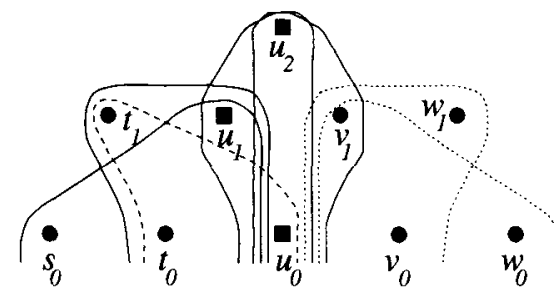

Fig. 15. 
Proof. If the D-triples are not present, then they can be added because anything in conflict with them was already in conflict with the $\mathbf{N}$-triples or the $\mathbf{V}$-triples, respectively. See Fig. 18.

Observation 3.8. A 0-edge belongs to at least one D-triple.

Proof. If $u_{0} v_{0}$ is not in a D-triple, then contracting $u_{0} v_{0}$ results in a $(1,1)$ reduction (one of two $\mathbf{H}$-triples containing $u_{0} v_{0}$ may be lost, no $\mathbf{N}$-triples may be lost because of Observation 3.7(i)).

\section{Observation 3.9. A 1-vertex belongs to at least one D-triple.}

Proof. Suppose $u_{1}$ is not in any D-triple. We can assume that $u_{1}$ is not in any $\mathbf{V}$-triple, for in that case, according to Observation 3.7(ii), a D-triple containing $u_{1}$ could be added. Likewise, $u_{1}$ cannot be in the $\mathbf{N}$-triples $\left(u_{0} u_{1} v_{1}\right)$ and $\left(v_{0} u_{1} v_{1}\right)$ (or $\left(t_{0} t_{1} u_{1}\right)$ and $\left(u_{0} t_{1} u_{1}\right)$ ), for Observation 3.7(i) implies that $u_{1}$ is in a D-triple. Thus, $u_{1}$ is in only two triples, the $\mathbf{N}$-triples $\left(u_{0} t_{1} u_{1}\right)$ and $\left(u_{0} u_{1} v_{1}\right)$. Then $t_{1} u_{1} v_{1}$ can be contracted into an edge resulting in a $(1,1)$ reduction. See Fig. 19.

Observation 3.10. A 0-edge belongs to at most three D-triples.

Proof. Let $u_{0} v_{0}$ belong to $\mathbf{D}$-triples with $t_{1}, u_{1}, v_{1}, w_{1}$. Then neither of the 1-edges $t_{1} u_{1}, u_{1} v_{1}, v_{1} w_{1}$ belong to a $\langle 2,2\rangle$-configuration, and the edge $u_{1} v_{1}$ is not in any $\mathrm{N}$-triple. See Fig. 20. Thus, the edge $u_{1} v_{1}$ can be contracted resulting in a $(1,1)$ reduction.

Observation 3.11. A 1-vertex belongs to at most two D-triples.

Proof. Vertex $u_{1}$ can belong to at most three D-triples because triples $\left(s_{0} t_{0} u_{1}\right)$ and $\left(v_{0} w_{0} u_{1}\right)$ would conflict. Suppose $u_{1}$ belongs to the three D-triples $\left(t_{0} u_{0} u_{1}\right),\left(u_{0} v_{0} u_{1}\right)$, and $\left(v_{0} w_{0} u_{1}\right)$. We consider three cases:

(i) No H-triple contains $u_{0} v_{0}$. Then one of the triples can be eliminated by contracting $u_{0} v_{0}$ into a vertex with a loss of one triple, a $(1,1)$ reduction. This may fail only if because of the contraction other triples are lost; but this can only happen for $\mathrm{N}$-triples using $u_{0}$ or $v_{0}$ and 1-edges $t_{1} u_{1}$ or $u_{1} v_{1}$. However, because of Observation 3.4 the only possible $\mathbf{N}$-triples are $\left(u_{0} t_{1} u_{1}\right)$ and $\left(v_{0} u_{1} v_{1}\right)$, and they remain different after contracting $u_{0} v_{0}$. See Fig. 21(i).

(ii) One $\mathbf{H}$-triple contains $u_{0} v_{0}$, say $\left(t_{0} u_{0} v_{0}\right)$. Note that $u_{1}$ is in at most one $\mathbf{N}$-triple, $\left(v_{0} u_{1} v_{1}\right)$. Then remove $u_{1}$ and contract $t_{0} u_{0} v_{0} w_{0}$ into an edge resulting in a $(3,5)$ reduction (or $(4,7)$ reduction if there is $u_{2}$ in a configuration $\left[v_{0} w_{0}, u_{1} v_{1}, u_{2}\right]$ ). See Fig. 21(ii) and the reduction in Fig. 22.

(iii) Two $\mathbf{H}$-triples contain $u_{0} v_{0},\left(t_{0} u_{0} v_{0}\right)$ and $\left(u_{0} v_{0} w_{0}\right)$. Note that $u_{1}$ is not in any $\mathrm{V}$ - or $\mathrm{N}$-triple. Then remove $u_{1}$ and contract $t_{0} u_{0} v_{0} w_{0}$ to an edge resulting in a $(3,5)$ reduction. See Fig. 21(iii). 


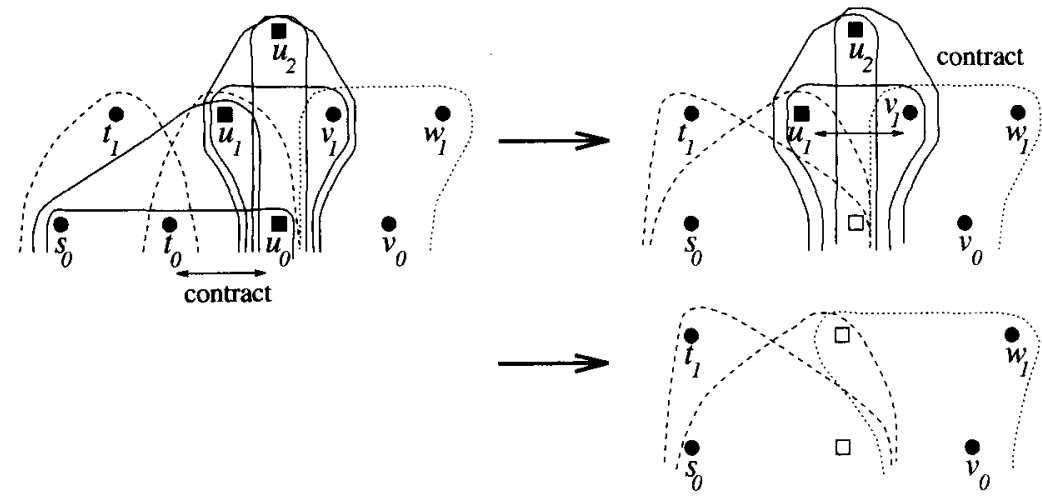

Fig. 16.
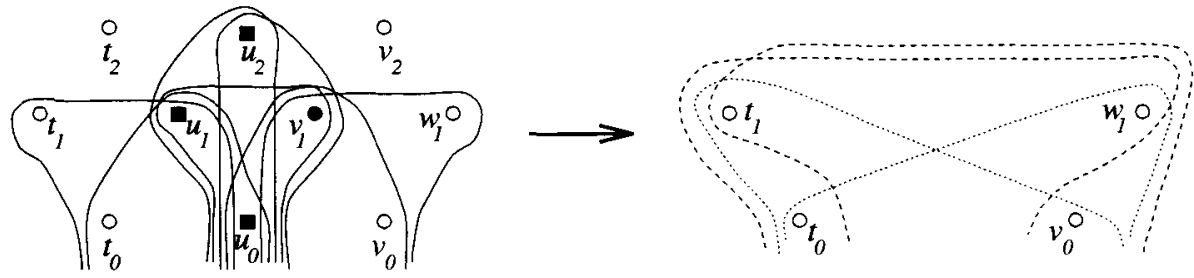

Fig. 17.
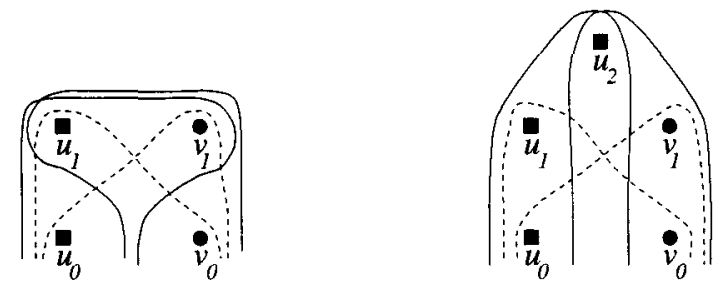

Fig. 18.

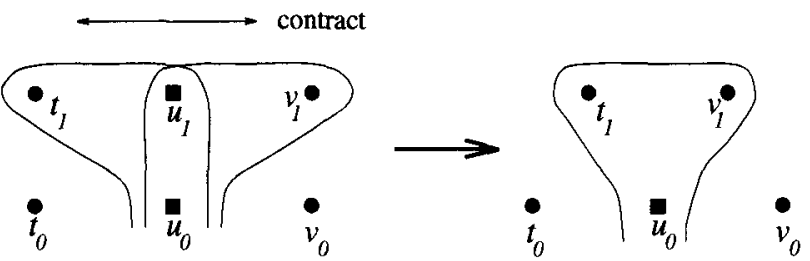

Fig. 19.

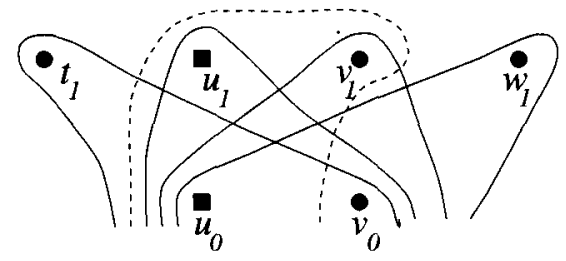

Fig. 20. 


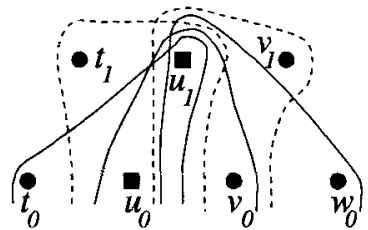

(i)

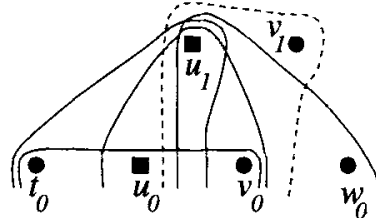

(ii)

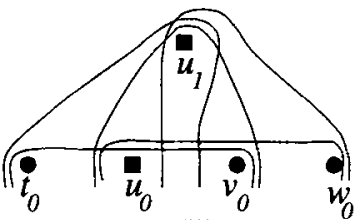

(iii)

Fig. 21.

Observation 3.12. Two adjacent 0-edges form D-triples with a common 1-vertex.

Proof. Suppose the statement is not true for $u_{0} v_{0}$ and $v_{0} w_{0}$, and consider the rightmost D-triple of $u_{0} v_{0}$ and the leftmost D-triple of $v_{0} w_{0}$. Because of Observation 3.9, the corresponding 1-vertices are consecutive, so let the triples be $\left(u_{0} v_{0} u_{1}\right)$ and $\left(v_{0} w_{0} v_{1}\right)$. If $\left(u_{0} v_{0} v_{1}\right)$ cannot be a triple, then either $\left(v_{0} \alpha u_{1}\right)$ or $\left(x_{0} v_{1} \beta\right)$ is present where $\alpha=t_{1}$ or $u_{2}$ and $\beta=y_{0}, w_{1}$, or $v_{2}$. See Fig. 23. However, $\beta \neq y_{0}$ or $v_{2}$ because then $v_{1}$ is in three D-triples. Similarly if $\left(v_{0} w_{0} u_{1}\right)$ cannot be a triple.

Thus, if neither $\left(u_{0} v_{0} v_{1}\right)$ nor $\left(v_{0} w_{0} u_{1}\right)$ can be a $\mathbf{D}$-triple, then either of the following two cases must hold:

(i) The triples $\left(t_{0} t_{1} u_{1}\right)$ and $\left(x_{0} v_{1} w_{1}\right)$ are present.

(ii) The triples $\left(v_{0} \alpha u_{1}\right)$ and $\left(v_{0} v_{1} \beta\right)$ are present, where $\alpha=t_{1}$ or $u_{2}$ and $\beta=w_{1}$ or $v_{2}$.

See Fig. 24. In case (i), contracting $u_{0} v_{0} w_{0}$ into an edge results in a $(1,1)$ reduction (the triple that may be lost is the $\mathbf{H}$-triple $\left.\left(u_{0} v_{0} w_{0}\right)\right)$.

In case (ii), note that $u_{1} v_{1}$ is not in a $\langle 2,2\rangle$-configuration, and that the triples $\left(t_{0} u_{0} u_{1}\right)$ and $\left(w_{0} x_{0} v_{1}\right)$ are not present because of Observation 3.4. Thus, removing $v_{0}, u_{1}, v_{1}$ (and $u_{2}$ and/or $v_{2}$ if they form $\langle 2,2\rangle$-configurations $\left[u_{0} v_{0}, t_{1} u_{1}, u_{2}\right]$ and $\left[v_{0} w_{0}, v_{1} w_{1}, v_{2}\right]$, respectively) results in a $(3,5)$ reduction $((4,7)$ or $(5,9)$, respectively). The five triples that may be lost are $\left(u_{0} v_{0} u_{1}\right),\left(v_{0} w_{0} v_{1}\right),\left(v_{0} u_{1} v_{1}\right),\left(u_{0} t_{1} u_{1}\right),\left(w_{0} v_{1} w_{1}\right)$. Note that four new triples may be introduced after removing the vertices; they replace the triples $\left(v_{0} t_{1} u_{1}\right)$, $\left(v_{0} v_{1} w_{1}\right),\left(u_{0} v_{0} t_{1}\right),\left(v_{0} w_{0} w_{1}\right)$. This is similar to the last case in the proof of Observation 3.6.
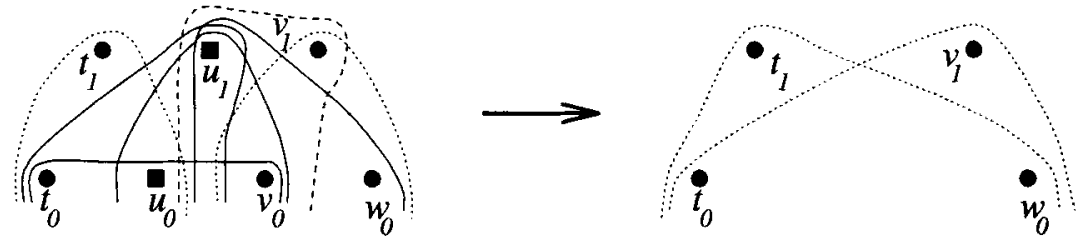

Fig. 22. 


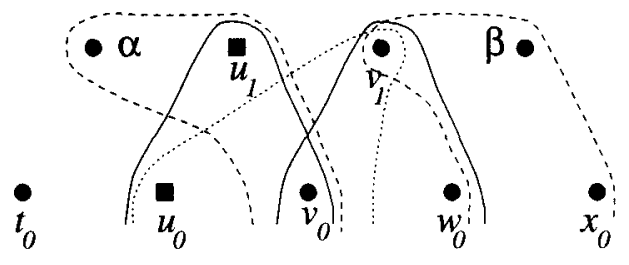

Fig. 23.

\section{Observation 3.13.}

(i) A 0-edge belongs to either two or three D-triples. A 0-edge that belongs to exactly three $\mathbf{D}$-triples does not belong to a $(2,2)$-configuration.

(ii) If $\left(u_{0} u_{1} v_{1}\right)$ is an $\mathbf{N}$-triple, then $\left(\alpha_{0} u_{0} u_{1}\right)$ and $\left(\alpha_{0} u_{0} v_{1}\right)$ are $\mathbf{D}$-triples where $\alpha_{0}=t_{0}$ or $\alpha_{0}=v_{0}$.

Proof. The first part of (i) follows from Observations 3.10,3.11, and 3.12 (the number of D-triples must be at least two). The second part of (i) is easily verified. (ii) is implied by several already existing constraints.

\section{Observation 3.14. There are no H-triples.}

Proof. First consider two $\mathbf{H}$-triples sharing a 0 -edge, say $\left(u_{0} v_{0} w_{0}\right)$ and $\left(v_{0} w_{0} x_{0}\right)$. Then by Observations $3.11,3.12$, and 3.13(i), there are vertices $u_{1}, v_{1}$ such that the following D-triples are present: $\left(u_{0} v_{0} u_{1}\right),\left(v_{0} w_{0} u_{1}\right),\left(v_{0} w_{0} v_{1}\right),\left(w_{0} x_{0} v_{1}\right)$. In particular, $v_{0} w_{0}$ belongs to exactly two $D$-triples, for the 1-vertex in a third one could be removed with a $(1,1)$ reduction or would contradict Observation 3.4. Also, neither $u_{1}$ nor $v_{1}$ are in a $\langle 2,2\rangle$-configuration because of Observations 3.7(ii) and 3.11. Edges $u_{0} v_{0}$ and $w_{0} x_{0}$ may be in three D-triples; let $\alpha_{1}$ and $\beta_{1}$ be the vertices forming the middle $\mathbf{D}$-triple containing $u_{0} v_{0}$ and $w_{0} x_{0}$, respectively, if they exist. See Fig. 25 (in that example there is no $\alpha_{1}$, and $\beta_{1}=w_{1}$ ). Then $\alpha_{1}, u_{1}, v_{1}, \beta_{1}$ can be substituted by a single 1-vertex, and $v_{0}, w_{0}$ removed, resulting in $(5,7),(4,6)$ (the case shown in the figure), or $(3,5)$ reduction depending on whether two, one, or none of $\alpha_{1}$ and $\beta_{1}$ exist.

Now, let $\tau_{1}=\left(u_{0} v_{0} w_{0}\right)$ be an $\mathbf{H}$-triple not sharing more than one vertex with another H-triple. By Observation 3.13, each of $u_{0} v_{0}$ and $v_{0} w_{0}$ is in two or three D-triples, and there is a vertex $u_{1}$ such that the triples $\left(u_{0} v_{0} u_{1}\right)$ and $\left(v_{0} w_{0} u_{1}\right)$ are present. Neither of $u_{0} v_{0}$ and $v_{0} w_{0}$ is in a $\langle 2,2\rangle$-configuration. Let $\alpha_{1}$ and $\beta_{1}$ be as before. See Fig. 26 (in

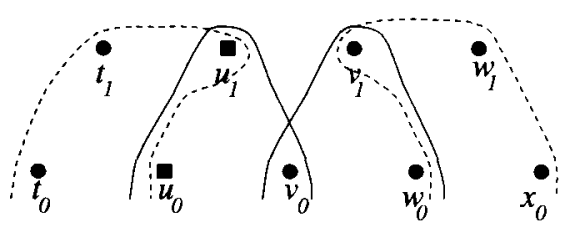

(i)

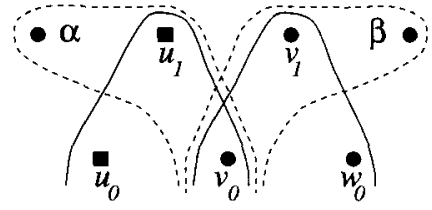

(ii)

Fig. 24. 
that example there is no $\alpha_{1}$ and $\beta_{1}=v_{1}$ ). Then $\alpha_{1}, u_{1}, \beta_{1}$ can be substituted by a single 1 -vertex, and $v_{0}$ removed, resulting in a $(3,4),(2,3)$ (the case shown in the figure), or $(1,1)$ reduction depending on whether two, one, or none of $\alpha_{1}$ and $\beta_{1}$ exist.

Note that we used Observation 3.13(ii) implicitly to restrict the possible $\mathbf{N}$-triples.

Observation 3.15. No two adjacent 0-edges can belong each to exactly two D-triples, or to exactly three $\mathbf{D}$-triples.

Proof. Let $u_{0} v_{0}$ have exactly three $\mathbf{D}$-triples, say with $u_{1}, v_{1}, w_{1}$. Then there is no $\langle 2,2\rangle$ configuration containing $u_{0} v_{0}$, and the only $\mathbf{N}$-triples containing $u_{1}, v_{1}, w_{1}$ are $\left(u_{0} u_{1} v_{1}\right)$ and $\left(v_{0} v_{1} w_{1}\right)$. Thus, a 0 -edge with three $\mathbf{D}$-triples contributes a total of five triples. On the other hand, a 0-edge with two D-triples contributes at least two triples; four triples if in a $\langle 2,2\rangle$-configuration, and at most six triples depending on the neighbor 0-edges (an $\mathbf{N}$-triple may conflict with the $\mathbf{N}$-triples of neighbor 0 -edges). See Fig. 27. If two adjacent 0 -edges each belongs to exactly two $\mathbf{D}$-triples, then they can be collapsed into one by removing two or three vertices (two if the 0 -edges are not in $\langle 2,2\rangle$-configurations), resulting in a $(2,3)$ or $(3,5)$ reduction. See Fig. 28 . If two adjacent 0 -edges each belongs to exactly three D-triples, then they can be collapsed into one by removing three vertices, resulting in a $(3,5)$-reduction. See Fig. 29.

This leaves as the only possibility a configuration in which 0 -edges alternate between having exactly two D-triples (and possibly a $\langle 2,2\rangle$-configuration) and having exactly three D-triples. Then two adjacent 0 -edges (and their corresponding 1 - and 2-vertices) can be removed, resulting in a $(5,9)$ reduction, or a $(6,11)$ reduction if there is a $\langle 2,2\rangle$ configuration. This last configuration is precisely the one shown in Fig. 7. This implies an upper bound $11 n / 6+C$ for $g_{3}^{\prime}(n)$ where $C$ is a constant.

\section{Lemma 3.16.}

(i) $g_{3}^{\prime}(n) \leq 11(n-2) / 6+\frac{1}{2}$ for $n \geq 3$.

(ii) $g_{3}^{\prime}(n) \leq 9(n-2) / 5+\frac{2}{5}$ for $n \geq 3$ if there is no $\langle\mathbf{2}, 2\rangle$-configuration.

Proof. The proof is by induction using the previous observations. A better multiplicative constant is obtained in case (ii) because $(6,11)$ reductions occur only if there are $\langle 2,2\rangle$-configurations. The constants $\frac{1}{2}$ and $\frac{2}{5}$ appear to take care of some particular configurations in the basis: in case (i), five vertices in a $\langle 2,2\rangle$-configuration with a total of six triples; in case (ii), four vertices (two in the 0-layer and two in the 1-layer) with four triples.

The proof of the upper bound in the following lemma is similar to that for $g_{2}(n)$. The optimal configuration is obtained by repeating the following basic configuration with an overlapping 0-edge: a 1-vertex forming $\mathbf{D}$-triples with three consecutive 0 -edges, and each two consecutive 0 -edges form an $\mathbf{H}$-triple.

Lemma 3.17. $g_{3}(n) \leq\lfloor 5 n / 3\rfloor$ for $n \geq 10$ if only $\mathbf{D}$ - and $\mathbf{H}$-triples are allowed. 


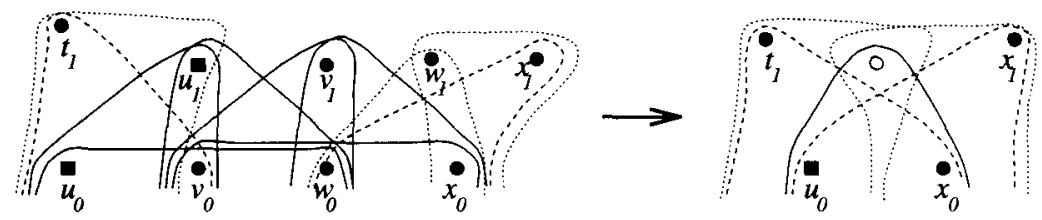

Fig. 25.

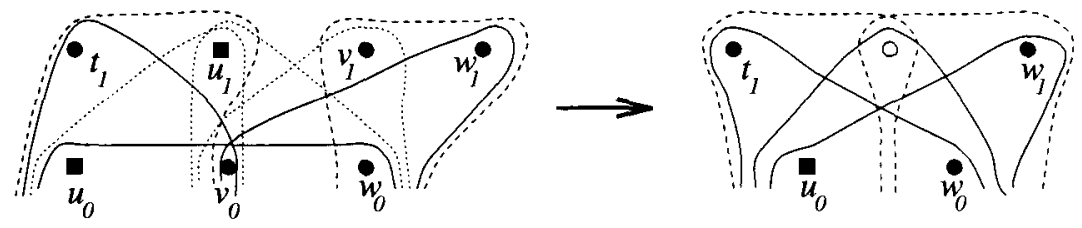

Fig. 26.
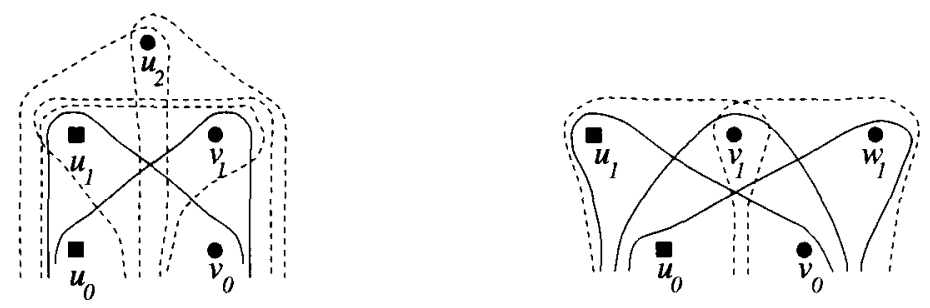

Fig. 27. 0-edges in exactly two and three D-triples.
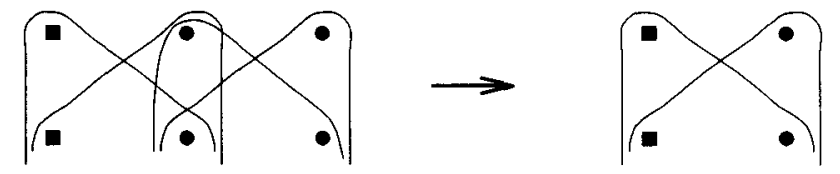

Fig. 28.

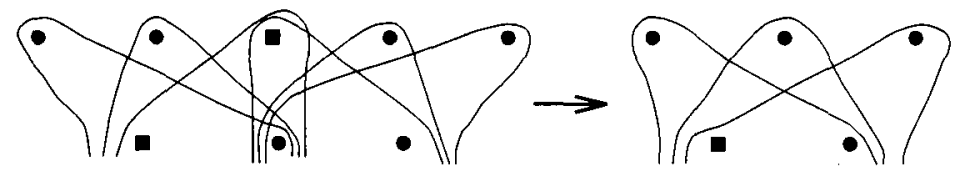

Fig. 29. 


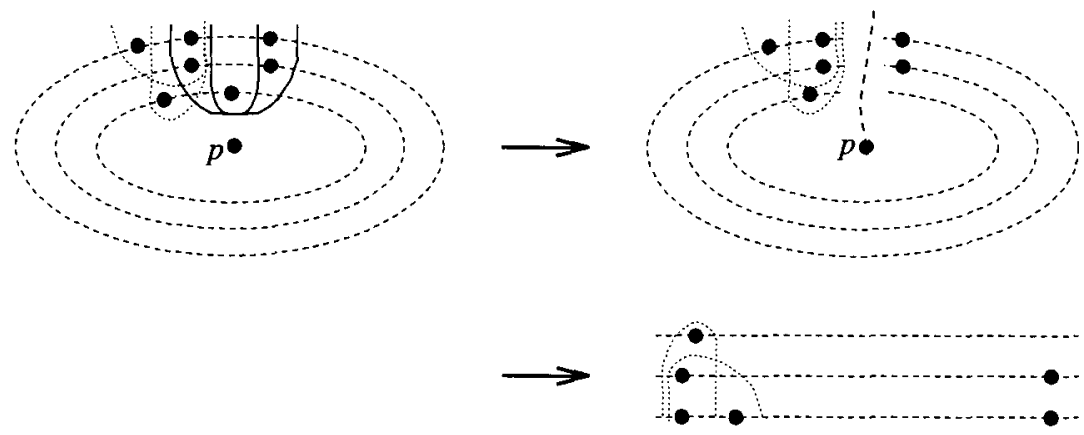

Fig. 30. Cutting open the arrangement.

Theorem 3.18. $\quad e_{3}(n) \leq g_{3}(n) \leq\lfloor 11 n / 6\rfloor+1$ for $n \geq 10$

Proof. Recall the constraint $n \geq 3 \cdot 3+1=10$ to obtain the layers with the point inside. A 2-vertex is in four $\mathbf{V}$-triples only if $n=5$, so this case is disregarded; it is in three $\mathbf{V}$ triples in a $\langle\mathbf{2}, 2\rangle^{\prime}$-configuration (see proof of Observation 3.2, and it is in two $\mathbf{V}$-triples in configurations $\langle 2,2\rangle,\langle\mathbf{1}, \mathbf{2}\rangle$, or $\langle\mathbf{2}, \mathbf{1}\rangle$ (without the no wrapping around condition, it may not be possible to reduce a $\langle 2,2\rangle^{\prime}$-configuration to a $\langle 2,2\rangle$-configuration). We consider the following cases:

(i) there is a 2-vertex $u_{2}$ in a $\langle 2,2\rangle^{\prime}$ - or $\langle 2,2\rangle$-configuration $\left[u_{0} v_{0}, u_{1} v_{1}, u_{2}\right]$; else

(ii) there is a 2-vertex $u_{2}$ in a $\langle\mathbf{1}, 2\rangle$-configuration $\left[u_{0}, u_{1} v_{1}, u_{2}\right]$; else

(iii) there is a 2-vertex $u_{2}$ in a $\langle 2, \mathbf{1}\rangle$-configuration; else

(iv) there is an $\mathrm{N}$-triple $\left(u_{0} u_{1} v_{1}\right)$; else

(v) there is a D-triple $\left(u_{0} v_{0} u_{1}\right)$; else

(vi) there are only $\mathbf{H}$-triples.

In case (i) the number of triples using only vertices $u_{2}, u_{1}, v_{1}, u_{0}, v_{0}$ is at most six. As illustrated in Fig. 30 the arrangement can be cut open: the six pseudolines are removed and the remaining arrangement is modified so that no pseudoline intersects a halfline from $p$ (shown dashed in the figure). Then Lemma 3.16(i) leads to the bound $\left(11((n-1)-2) / 6+\frac{1}{2}\right)+6=11 n / 6+1$ (the open configuration has $n-1$ vertices and six triples were removed). We proceed similarly in the other cases: the arrangement can be cut open and using Lemmas 3.16 and 3.17 we obtain the bounds $\left(9(n-2) / 5+\frac{2}{5}\right)+3=$ $9 n / 5-\frac{1}{5}$ for cases (ii) and (iii), $\left(9((n+2)-2) / 5+\frac{2}{5}\right)+0=9 n / 5+\frac{2}{5}$ for case (iv) (in this case no triples are lost as a result of cutting open the arrangement), $5 n / 3$ for case (v), and $n$ for case (vi) (no need to cut open the arrangement in the last two cases). Overall, we obtain the bound $11 n / 6+1$.

\section{Acknowledgments}

I thank Herbert Edelsbrunner for encouraging me to write this note, and for suggestions to improve the presentation, and to an anonymous referee for a very thorough review. 


\section{References}

1. H. Edelsbrunner, Algorithms in Combinatorial Geometry, Springer-Verlag, Heidelberg, 1987.

2. H. Edelsbrunner and G. Stöckl, The number of extreme pairs of finite point-sets in Euclidean spaces, J. Combin. Theory Ser. A 43 (1986), 344-349.

3. J. E. Goodman, Proof of a conjecture of Burr, Grünbaum, and Sloane, Discrete Math. 32 (1980), 27-35.

4. J. E. Goodman and R. Pollack, Allowable sequences and order types in discrete and computational geometry, in New Trends in Discrete and Computational Geometry, J. Pach, ed., pp. 103-134, Springer-Verlag, New York, 1993.

5. J. Snoeyink and J. Hershberger, Sweeping arrangements of curves, in Discrete and Computational Geometry: Papers from the DIMACS Special Year, J. E. Goodman, R. Pollack, and W. Steiger, eds., pp. 309-349, AMS, Providence, RI, and ACM, New York, 1991.

6. G. Stöckl, Gesammelte und neue Ergebnisse über extreme $k$-Mengen für ebene Punktmengen, Diplomarbeit, Institutes for Information Processing, Technical University of Graz, Graz, 1984.

Received May 15, 1994, and in revised form August 3, 1995. 